Received by the editors: August 3, 2020; Accepted: March 3, 2021

\title{
THE EXISTENCE OF THE BOUNDED SOLUTIONS OF A SECOND ORDER NONHOMOGENEOUS NONLINEAR DIFFERENTIAL EQUATION
}

\author{
Mehtap LAFCI BUYUKKAHRAMAN \\ Department of Mathematics, Faculty of Arts and Sciences, \\ Uşak University, Uşak, 64200, TURKEY
}

\begin{abstract}
In this paper, we consider a second order nonlinear differential equation and establish two new theorems about the existence of the bounded solutions of a second order nonlinear differential equation. In these theorems, we use different Lyapunov functions with different conditions but we get the same result. In addition, two examples are given to support our results with some figures.
\end{abstract}

\section{INTROduCtion}

For more than sixty years, a great deal of work has been done by various authors to investigate the autonomous and non-autonomous second order nonlinear ordinary differential equations (ODEs) ( [1]- [5], 7]- 14], [16], 17], 19] ) and references cited therein.

In investigating the qualitative properties of solutions for second order ODEs, the fixed point method, perturbation theory, variations of parameter formulas, etc. have been used to get information without solving the equations. Moreover, in some of these works, the authors have been studied the Lyapunov direct or second method by constructing different Lyapunov functions or using existing Lyapunov functions.

As far as we know, it should be noted in the relevant literature that so far, the second method of Lyapunov is the most effective tool for studying qualitative

2020 Mathematics Subject Classification. Primary 34C11; Secondary 34D05.

Keywords and phrases. Nonlinear differential equation, second order, boundedness, the Lyapunov second method.

mehtap.lafci@usak.edu.tr

(D) 0000-0001-9813-8195.

(C)2021 Ankara University Communications Faculty of Sciences University of Ankara-Series A1 Mathematics and Statistics 
features of nonlinear higher order equations without getting solutions of the equations. This method needs the creation of an appropriate function or functionality that gives concrete results for the problem being studied.

In 1995, Meng [6] dealt with the ordinary linear differential equation of second order

$$
x^{\prime \prime}(t)+p(t) x^{\prime}(t)+\left[q_{1}(t)+q_{2}(t)\right] x(t)=f(t),
$$

and in 2002, Yuangong and Fanwei [18 considered the second order time lag nonlinear differential equation

$$
\left(r(t) x^{\prime}(t)\right)^{\prime}+p(t) x^{\prime}(t)+\left[q_{1}(t)+q_{2}(t)\right] x(t)=f(t, x(t)) .
$$

The authors got some interesting results on the boundedness and square integrability of solutions of the ODEs.

In 2019, Tunç and Mohammed 15 considered two different models for nonlinear of second order

$$
x^{\prime \prime}(t)+p(t) g\left(x^{\prime}\right)+q_{1}(t) h(x)+q_{2}(t) x=f\left(t, x, x^{\prime}\right)
$$

and

$$
x^{\prime \prime}(t)+\Phi\left(t, x, x^{\prime}\right)+q_{1}(t) x+q_{2}(t) \theta(x)=q\left(t, x, x^{\prime}\right) .
$$

They investigate asymptotic boundedness of solutions of the ODEs as $t \rightarrow \infty$.

In this paper, motivated by the work of Tunc and Mohammed [15], we deal with the following second order nonlinear differential equation:

$$
x^{\prime \prime}+f\left(t, x, x^{\prime}\right)+q_{1}(t) \varphi(x)+q_{2}(t) \psi(x)=g\left(t, x, x^{\prime}\right),
$$

where $x \in \mathbb{R}=(-\infty, \infty), t \in \mathbb{R}^{+}=[0, \infty) . f \in C^{1}\left(\mathbb{R}^{+} \times \mathbb{R}^{2}, \mathbb{R}\right), q_{1}, q_{2} \in C^{1}\left(\mathbb{R}^{+}, \mathbb{R}\right)$, $\varphi, \psi \in C^{1}(\mathbb{R}, \mathbb{R}), g \in C\left(\mathbb{R}^{+} \times \mathbb{R}^{2}, \mathbb{R}\right)$ and $f(t, x, 0)=0, \varphi(0)=0, \psi(0)=0$. Under the assumptions, the existence of the solutions of Eq. (1) is guaranteed. In addition, we assume that the functions $f, \varphi, \psi$ and $g$ fulfill the Lipschitz condition with respect to $x$ and its derivative $x^{\prime}$. So, the solutions of Eq. (1) are uniqueness.

Eq. (1) can be written as

$$
\begin{aligned}
x^{\prime} & =y \\
y^{\prime} & =-f\left(t, x, x^{\prime}\right)-q_{1}(t) \varphi(x)-q_{2}(t) \psi(x)+g\left(t, x, x^{\prime}\right) .
\end{aligned}
$$

Let

$$
\begin{aligned}
& \varphi^{*}(x)=\left\{\begin{array}{cc}
x^{-1} \varphi(x), & x \neq 0 \\
\varphi^{\prime}(0), & x=0,
\end{array}\right. \\
& \psi^{*}(x)=\left\{\begin{array}{cc}
x^{-1} \psi(x), & x \neq 0 \\
\psi^{\prime}(0), & x=0
\end{array}\right.
\end{aligned}
$$

and

$$
f^{*}(t, x, y)=\left\{\begin{array}{cc}
y^{-1} f(t, x, y), & y \neq 0 \\
f_{y}^{\prime}(t, x, 0), & y=0
\end{array}\right.
$$




\section{Main Results}

The following assumptions are needed to formulate our main results.

(A1) $f(t, x, 0)=0, y^{-1} f(t, x, y) \geq f_{0} \geq 1$ for all $t \in \mathbb{R}^{+}, x \in \mathbb{R}, y \in \mathbb{R}-\{0\}$.

(A2) $\varphi(0)=0, x^{-1} \varphi(x) \geq \varphi_{0} \geq 1$ for all $x \in \mathbb{R}-\{0\}$.

(A3) $\psi(0)=0, x^{-2} \psi^{2}(x) \leq 1$ for all $x \in \mathbb{R}-\{0\}$.

(A4) $\psi(0)=0, x^{-1} \psi(x) \geq \psi_{0} \geq 1$ for all $x \in \mathbb{R}-\{0\}$.

(A5) $q_{1}(t)>0, q_{2}(t)>0, q_{1}^{\prime}(t)>0, \forall t \in \mathbb{R}^{+}$.

(A6) The functions $g_{1}(t), \Theta(t), h(t)$ are continuous such that

$$
\begin{gathered}
|g(t, x, y)| \leq\left|g_{1}(t)\right|, \forall t \in \mathbb{R}^{+}, \forall x, y \in \mathbb{R}, \\
\Theta(t)=\frac{1}{2}\left(q_{1}^{\prime}(t)+2 q_{1}(t)\right), \forall t \in \mathbb{R}^{+}, \\
\int_{a}^{\infty} \frac{q_{2}^{2}(s)}{h^{2}(s) \Theta(s)} d s<\infty, \quad \int_{a}^{\infty} \frac{g_{1}^{2}(s)}{\Theta(s)} d s<\infty, \\
h^{2}(t) \geq 1, \quad \forall t \in \mathbb{R}^{+} .
\end{gathered}
$$

Theorem 1. If the conditions $(A 1),(A 2),(A 3),(A 5)$ and $(A 6)$ hold, any solution of Eq. (1) satisfies

$$
|x(t)| \leq O(1),\left|\frac{d x}{d t}\right| \leq O\left(\sqrt{q_{1}(t)}\right), t \rightarrow \infty .
$$

Proof. We establish the following Lyapunov function because we use the Lyapunov second method

$$
V(x, y)=2 \int_{0}^{x} \varphi(\zeta) d \zeta+\frac{1}{q_{1}(t)} y^{2} .
$$

From $(A 1),(A 2),(A 5)$ and $(A 6)$, we get $V(x, y)=0$ if and only if $x=0$ and $y=0$. From $(A 2)$ and $q_{1}(t)>0$, we have

$$
V(x, y) \geq x^{2}+\frac{1}{q_{1}(t)} y^{2} \geq 0 .
$$

Differentiating the Lyapunov function $V$ in (4) along the solutions of the system (2) and using ( $A 1)$, we obtain

$$
\begin{aligned}
\frac{d}{d t} V & =-\frac{q_{1}^{\prime}(t)}{q_{1}^{2}(t)} y^{2}-\frac{2}{q_{1}(t)} y f(t, x, y)-2 \frac{q_{2}(t)}{q_{1}(t)} y \psi(x)+\frac{2}{q_{1}(t)} y g(t, x, y) \\
& \leq-\frac{q_{1}^{\prime}(t)}{q_{1}^{2}(t)} y^{2}-\frac{2}{q_{1}(t)} y^{2}-2 \frac{q_{2}(t)}{q_{1}(t)} y \psi(x)+\frac{2}{q_{1}(t)} y g(t, x, y) \\
& =-\frac{2}{q_{1}^{2}(t)}\left[\frac{1}{2} q_{1}^{\prime}(t)+q_{1}(t)\right] y^{2}-2 \frac{q_{2}(t)}{q_{1}(t)} y \psi(x)+\frac{2}{q_{1}(t)} y g(t, x, y) .
\end{aligned}
$$

Since

$$
\Theta(t)=\frac{1}{2}\left(q_{1}^{\prime}(t)+2 q_{1}(t)\right)
$$


we have

$$
\frac{d}{d t} V \leq-\frac{2 \Theta(t)}{q_{1}^{2}(t)} y^{2}-2 \frac{q_{2}(t)}{q_{1}(t)} y \psi(x)+\frac{2}{q_{1}(t)} y g(t, x, y)
$$

We assume that $a>0, b, x \in \mathbb{R}$. If we use the inequality

$$
-a x^{2}+b x \leq-\frac{a}{2} x^{2}+\frac{b^{2}}{2 a}
$$

to the terms

$$
-\frac{2 \Theta(t)}{q_{1}^{2}(t)} y^{2}+\frac{2}{q_{1}(t)} y g(t, x, y)
$$

and from $(A 5),(A 6)$, we get

$$
\frac{d}{d t} V \leq-\frac{\Theta(t)}{q_{1}^{2}(t)} y^{2}-2 \frac{q_{2}(t)}{q_{1}(t)} y \psi(x)+\frac{g_{1}^{2}(t)}{\Theta(t)} .
$$

Let

$$
W(x, y)=-\frac{\Theta(t)}{q_{1}^{2}(t)} y^{2}-2 \frac{q_{2}(t)}{q_{1}(t)} y \psi(x) .
$$

Rearranging $W(x, y)$, we have

$$
W(x, y)=-\frac{\Theta(t)}{q_{1}^{2}(t)}\left[h(t) y+\frac{q_{1}(t) q_{2}(t)}{h(t) \Theta(t)} \psi(x)\right]^{2}+\frac{q_{2}^{2}(t)}{h^{2}(t) \Theta(t)} \psi^{2}(x)+\frac{\Theta(t)}{q_{1}^{2}(t)}\left(h^{2}(t)-1\right) y^{2} .
$$

Since the first term of $W(x, y)$ is negative, it is clear that

$$
W(x, y) \leq \frac{q_{2}^{2}(t)}{h^{2}(t) \Theta(t)} \psi^{2}(x)+\frac{\Theta(t)}{q_{1}^{2}(t)}\left(h^{2}(t)-1\right) y^{2} .
$$

From (7) and (8)

$$
\frac{d}{d t} V \leq \frac{q_{2}^{2}(t)}{h^{2}(t) \Theta(t)} \psi^{2}(x)+\frac{\Theta(t)}{q_{1}^{2}(t)}\left(h^{2}(t)-1\right) y^{2}+\frac{g_{1}^{2}(t)}{\Theta(t)} .
$$

We assume that

$$
\frac{q_{2}^{2}(t)}{h^{2}(t) \Theta(t)}=\frac{\Theta(t)}{q_{1}(t)}\left(h^{2}(t)-1\right)
$$

Hence

$$
h^{2}(t)=\frac{\Theta^{2}(t)+\sqrt{\Theta^{4}(t)+4 q_{1}(t) q_{2}^{2}(t) \Theta^{2}(t)}}{2 \Theta^{2}(t)} .
$$

So, it can be seen that $h^{2}(t) \geq 1$ for $t \in \mathbb{R}^{+}$. Thus, we obtain

$$
W(x, y) \leq \frac{q_{2}^{2}(t)}{h^{2}(t) \Theta(t)}\left[\psi^{2}(x)+\frac{1}{q_{1}(t)} y^{2}\right] .
$$

From $(9)$ and $(10)$

$$
\frac{d}{d t} V \leq \frac{q_{2}^{2}(t)}{h^{2}(t) \Theta(t)}\left[\psi^{2}(x)+\frac{1}{q_{1}(t)} y^{2}\right]+\frac{g_{1}^{2}(t)}{\Theta(t)} .
$$


Also, from (A3), we know that

$$
\psi^{2}(x)+\frac{1}{q_{1}(t)} y^{2} \leq x^{2}+\frac{1}{q_{1}(t)} y^{2} \leq V(t) .
$$

And applying the inequality to (11), we can derive

$$
\frac{d}{d t} V-\frac{q_{2}^{2}(t)}{h^{2}(t) \Theta(t)} V \leq \frac{g_{1}^{2}(t)}{\Theta(t)} .
$$

Multiplying the inequality by

$$
\exp \left(-\int_{t_{0}}^{t} \frac{q_{2}^{2}(s)}{h^{2}(s) \Theta(s)} d s\right)
$$

and integrating this inequality from $t_{0}$ to $t$, we get

$$
V(t) \leq V\left(t_{0}\right) \exp \left(\int_{t_{0}}^{t} \frac{q_{2}^{2}(s)}{h^{2}(s) \Theta(s)} d s\right)+\int_{t_{0}}^{t}\left[\frac{g_{1}^{2}(s)}{\Theta(s)} \exp \left(\int_{s}^{t} \frac{q_{2}^{2}(\eta)}{h^{2}(\eta) \Theta(\eta)} d \eta\right)\right] d s .
$$

Hence we can take

$V(t) \leq V\left(t_{0}\right) \exp \left(\int_{t_{0}}^{\infty} \frac{q_{2}^{2}(s)}{h^{2}(s) \Theta(s)} d s\right)+\int_{t_{0}}^{\infty}\left[\frac{g_{1}^{2}(s)}{\Theta(s)} \exp \left(\int_{s}^{\infty} \frac{q_{2}^{2}(\eta)}{h^{2}(\eta) \Theta(\eta)} d \eta\right)\right] d s$.

Because of $(A 6)$, we can assume that

$$
V\left(t_{0}\right) \exp \left(\int_{t_{0}}^{\infty} \frac{q_{2}^{2}(s)}{h^{2}(s) \Theta(s)} d s\right)+\int_{t_{0}}^{\infty}\left[\frac{g_{1}^{2}(s)}{\Theta(s)} \exp \left(\int_{s}^{\infty} \frac{q_{3}^{2}(\eta)}{h^{2}(\eta) \Theta(\eta)} d \eta\right)\right] d s=A,
$$

where $A>0, A \in \mathbb{R}$. So, we have

$$
V(t) \leq A
$$

and

Therefore, we find

$$
x^{2}+\frac{1}{q_{1}(t)} y^{2} \leq V(t) \leq A .
$$

Hence

$$
|x(t)| \leq \sqrt{A}, \quad|y(t)| \leq \sqrt{A q_{1}(t)} .
$$

$$
|x(t)| \leq O(1), \quad|y(t)| \leq O\left(\sqrt{q_{1}(t)}\right), \quad t \rightarrow \infty .
$$

The result of the following theorem is the same as the result of Theorem 1 but we use different Lyapunov function and some different conditions in Theorem 2 .

Theorem 2. If the conditions $(A 1),(A 2),(A 4),(A 5)$ and $(A 6)$ hold, any solution of Eq. (1) satisfies

$$
|x(t)| \leq O(1), \quad\left|\frac{d x}{d t}\right| \leq O\left(\sqrt{q_{1}(t)}\right), \quad t \rightarrow \infty .
$$


Proof. We determine the Lyapunov function as follows

$$
V(x, y)=2 \int_{0}^{x}\left[\varphi(\zeta)+\frac{q_{2}(t)}{q_{1}(t)} \psi(\zeta)\right] d \zeta+\frac{1}{q_{1}(t)} y^{2} .
$$

From $(A 1),(A 2),(A 4),(A 5)$ and $(A 6)$, we get $V(x, y)=0$ if and only if $x=0$ and $y=0$. From $(A 2),(A 4), q_{1}(t)>0$ and $q_{2}(t)>0$, we have

$$
V(x, y) \geq\left(1+\frac{q_{2}(t)}{q_{1}(t)}\right) x^{2}+\frac{1}{q_{1}(t)} y^{2} \geq 0 .
$$

Differentiating the Lyapunov function $V$ in 12 along the solutions of the system (2) and using (A1), we find

$$
\begin{aligned}
\frac{d}{d t} V & =-\frac{2}{q_{1}(t)} y f(t, x, y)+\frac{2}{q_{1}(t)} y g(t, x, y)-\frac{q_{1}^{\prime}(t)}{q_{1}^{2}(t)} y^{2} \\
& \leq-\frac{2 y^{2}}{q_{1}(t)}+\frac{2}{q_{1}(t)} y g(t, x, y)-\frac{q_{1}^{\prime}(t)}{q_{1}^{2}(t)} y^{2} \\
& =-\frac{2}{q_{1}^{2}(t)}\left[\frac{1}{2} q_{1}^{\prime}(t)+q_{1}(t)\right] y^{2}+\frac{2}{q_{1}(t)} y g(t, x, y) .
\end{aligned}
$$

Defining $\Theta(t)$ as in (5), we have

$$
\frac{d}{d t} V \leq-\frac{2 \Theta(t)}{q_{1}^{2}(t)} y^{2}+\frac{2}{q_{1}(t)} y g(t, x, y)
$$

Let $a>0, b, x \in \mathbb{R}$. From the inequality (6) and $(A 6)$, we get

$$
\frac{d}{d t} V \leq-\frac{\Theta(t)}{q_{1}^{2}(t)} y^{2}+\frac{g_{1}^{2}(t)}{\Theta(t)} .
$$

Since the first term of the inequality is negative, we can write

$$
\frac{d}{d t} V \leq \frac{g_{1}^{2}(t)}{\Theta(t)} .
$$

Integrating this inequality from $t_{0}$ to $t$, we get

$$
V(t) \leq V\left(t_{0}\right)+\int_{t_{0}}^{t} \frac{g_{1}^{2}(s)}{\Theta(s)} d s .
$$

Hence we can take

$$
V(t) \leq V\left(t_{0}\right)+\int_{t_{0}}^{\infty} \frac{g_{1}^{2}(s)}{\Theta(s)} d s .
$$

Because of $(A 6)$, we can assume that

$$
V\left(t_{0}\right)+\int_{t_{0}}^{\infty} \frac{g_{1}^{2}(s)}{\Theta(s)} d s=B, B>0, B \in \mathbb{R} .
$$

So, we have

$$
V(t) \leq B
$$


From $(A 2),(A 4)$ and $(A 5)$, we know that

$$
x^{2}+\frac{1}{q_{1}(t)} y^{2} \leq V(t) \leq B .
$$

Therefore, we find

$$
|x(t)| \leq \sqrt{B}, \quad|y(t)| \leq \sqrt{B q_{1}(t)}
$$

Hence

$$
|x(t)| \leq O(1),|y(t)| \leq O\left(\sqrt{q_{1}(t)}\right), t \rightarrow \infty .
$$

Remark 3. If it is taken $f\left(t, x, x^{\prime}\right)=p(t) g\left(x^{\prime}\right)$ and $\psi(x)=x$ in Eq. (1) or $\varphi(x)=x$ in Eq. (1), Theorem 1 or Theorem 2 in [15] is obtained, respectively.

\section{EXAmples}

Example 4. As a special case of Eq. (1), we consider the following second order nonlinear $O D E$

$$
x^{\prime \prime}+6 x^{\prime}+x^{\prime} e^{-t-x^{2}}+5 e^{3 t}(5+\sin x) x+2 e^{2 t}\left(1-e^{-x^{2}}\right) x=\frac{\cos t}{e^{3 t}\left(1+2 e^{x^{4}}\right)}
$$

or

$$
\begin{aligned}
& x^{\prime}=y \\
& y^{\prime}=-6 x^{\prime}-x^{\prime} e^{-t-x^{2}}-5 e^{3 t}(5+\sin x) x-2 e^{2 t}\left(1-e^{-x^{2}}\right) x+\frac{\operatorname{cost}}{e^{3 t}\left(1+2 e^{x^{4}}\right)} .
\end{aligned}
$$

It is clear that the conditions $(A 1),(A 2),(A 3),(A 5)$ and $(A 6)$ are satisfied. So, from Theorem 1, all solutions of Eq. 13) satisfy

$$
|x(t)| \leq O(1),\left|\frac{d x}{d t}\right| \leq O\left(\sqrt{5} e^{3 t}\right), t \rightarrow \infty
$$

as shown in Fig. 1 obtained by using the adaptive MATLAB solver ode45.

Example 5. Taking $f\left(t, x, x^{\prime}\right)=5 x^{\prime} e^{t} \sin ^{2} x, q_{1}(t)=2 e^{3 t}, \varphi(x)=x e^{x^{2}}, q_{2}(t)=$ $5 e^{4 t}, \psi(x)=(3+\sin x) x$ and $g\left(t, x, x^{\prime}\right)=\frac{\sin x^{\prime}}{e^{6 t}\left(2+e^{x^{2}}\right)}$ in Eq. G1, we get the following second order nonlinear ODE

$$
x^{\prime \prime}+5 x^{\prime} e^{t} \sin ^{2} x+2 e^{3 t} x e^{x^{2}}+5 e^{4 t}(3+\sin x) x=\frac{\sin x^{\prime}}{e^{6 t}\left(2+e^{x^{2}}\right)}
$$

or

$$
\begin{aligned}
& x^{\prime}=y \\
& y^{\prime}=-5 x^{\prime} e^{t} \sin ^{2} x-2 e^{3 t} x e^{x^{2}}-5 e^{4 t}(3+\sin x) x+\frac{\sin x^{\prime}}{e^{6 t}\left(2+e^{x^{2}}\right)} .
\end{aligned}
$$




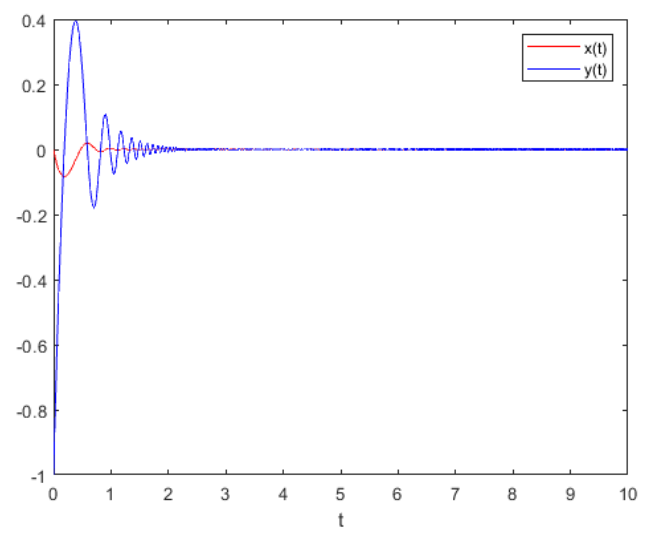

Figure 1. The solution of Eq. 13 with the initial conditions $x(0)=0, y(0)=-1$ in $t \in[0,10]$.

It is clear that the conditions $(A 1),(A 2),(A 4),(A 5)$ and $(A 6)$ are satisfied. So, from Theorem 2, all solutions of Eq. (14) satisfy

$$
|x(t)| \leq O(1), \quad\left|\frac{d x}{d t}\right| \leq O\left(\sqrt{2} e^{-3 t}\right), \quad t \rightarrow \infty
$$

as shown in Fig. 2 obtained by using the adaptive MATLAB solver ode45.

\section{Conclusion}

We have presented a new second order nonlinear differential equation (1) to study the existence of the bounded solutions of the equation by using the Lyapunov direct or second method. Additionally, we give two examples to support our main results. Also, MATLAB has been used to draw two figures. Fig. 1 in first example shows the solution $(x(t), y(t))$ of Eq. (13) with the initial conditions $x(0)=0, y(0)=-1$ in $t \in[0,10]$. The solution is bounded since the conditions of Theorem 1 are satisfied. Fig. 2 exemplifies the solution $(x(t), y(t))$ of Eq. (14) with the initial conditions $x(0)=1, y(0)=0$ in $t \in[0,7]$. The solution is bounded since the conditions of Theorem 2 are satisfied. Moreover, taking $f\left(t, x, x^{\prime}\right)=p(t) g\left(x^{\prime}\right)$ and $\psi(x)=x$ or $\varphi(x)=x$ in Eq. (1), Theorem 1 or Theorem 2 in [15] is gotten, respectively. So, Eq. (1) is a generalization of Eq. (6) and Eq. (7) in [15].

Declaration of Competing Interest The author has no competing interest to declare. 

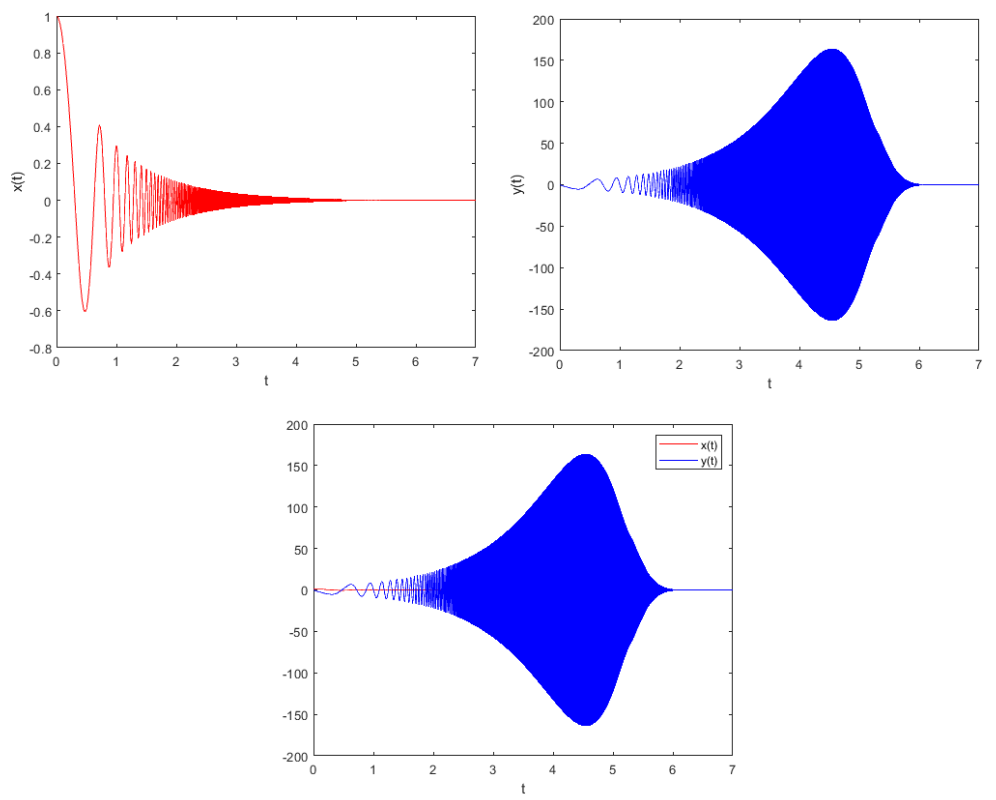

Figure 2. The solution of Eq. (14) with the initial conditions $x(0)=1, y(0)=0$ in $t \in[0,7]$.

\section{REFERENCES}

[1] Ademola, A., Boundedness and stability of solutions to certain second order differential equations, Differential Equations and Control Processes, 2015(3) (2015), 38-50.

[2] Alaba, J., Ogundare, B., On stability and boundedness properties of solutions of certain second order non-autonomous nonlinear ordinary differential equation, Kragujevac Journal of Mathematics, 39(2) (2015), 255-266/https://doi.org/10.5937/kgjmath1502255a

[3] Guzmán, P. M., Napoles Valdes, J. E., Lugo, L. M., On the boundedness of some nonlinear differential equation of second order, International Journal of Mathematics and its Applications, 3(4-E) (2015), 23-31.

[4] Jones, Jr, J., On the asymptotic stability of certain second order nonlinear differential equations, SIAM Journal on Applied Mathematics, 14(1) (1966), 16-22 https://doi.org/10. $1137 / 0114002$

[5] Korkmaz, E., Tunç, C., Convergence to non-autonomous differential equations of second order, Journal of the Egyptian Mathematical Society, 23(1) (2015), 27-30. https://doi.org/ $10.1016 / j \cdot$ joems.2014.02.009

[6] Meng, F., Quadratic integrability and boundedness of solutions to second-order inhomogeneous linear differential equations, J. Syst. Sci. Math. Sci., 15(1) (1995), 50-57.

[7] Mustafa, O. G., Tunç, C., Asymptotically linear solutions of differential equations via lyapunov functions, Applied Mathematics and Computation, 215(8) (2009), 3076-3081. https: //doi.org/10.1016/j.amc.2009.09.059 
[8] Nápoles Valdes, J. E., A note on the qualitative behavior of some second order nonlinear equation, Applications and Applied Mathematics, 8(2) (2013), 767-776.

[9] Qarawani, M. N., Boundedness and asymptotic behaviour of solutions of a second order nonlinear differential equation, Journal of Mathematics Research, 4(3) (2012), 121-127. https: //doi.org/10.5539/jmr.v4n3p121

[10] Tunç, C., A note on boundedness of solutions to a class of non-autonomous differential equations of second order, Applicable Analysis and Discrete Mathematics, 4(2) (2010), 361372. https://doi.org/10.2298/aadm100601026t

[11] Tunç, C., On the boundedness of solutions of a non-autonomous differential equation of second order, Sarajevo Journal of Mathematics, 7(19) (2011), 19-29.

[12] Tunç, C., Stability and boundedness of solutions of non-autonomous differential equations of second order, Journal of Computational Analysis and Applications, 13(6) (2011).

[13] Tunç, C., A note on the bounded solutions to $x^{\prime \prime}+c\left(t, x, x^{\prime}\right)+q(t) b(x)=f(t)$, Applied Mathematics and Information Sciences, 8(1) (2014), 393-399. https://doi.org/10.12785/ amis/080149

[14] Tunç, C., Altun, M., On the integrability of solutions of non-autonomous differential equations of second order with multiple variable deviating arguments, Journal of Computational Analysis and Applications, 14(1) (2012), 899-908.

[15] Tunç, C., Mohammed, S. A., On the asymptotic analysis of bounded solutions to nonlinear differential equations of second order, Advances in Difference Equations, 2019(1) (2019), 1-19. https://doi.org/10.1186/s13662-019-2384-x

[16] Tunç, C., Tunç, E., On the asymptotic behavior of solutions of certain second-order differential equations, Journal of the Franklin Institute, 344(5) (2007), 391-398. https://doi.org/ $10.1016 / j \cdot j$ franklin.2006.02.011

[17] Tunç, C., Tunç, O., A note on the stability and boundedness of solutions to non-linear differential systems of second order, Journal of the Association of Arab Universities for Basic and Applied Sciences, 24(1) (2017), 169-175. https://doi.org/10.1016/j.jaubas.2016.12. 004

[18] Yuangong, S., Fanwei, M., Quadratic integrability and boundedness for the solutions of second order nonlinear differential equations, Annals of Differential Equations, 18(1) (2002), 58-64.

[19] Zhao, L.-Q., On global asymptotic stability for a class of second order differential equations, Adv. Math., 35(3) (2006), 378-384. 